\title{
Bariatric surgery patient: is the patient a well- understood universe?
}

\section{Opinion}

Having clear our role as a physician in different scenarios out the hospital, at least in one opportunity a conversation about weight loss and obesity has occurred. We almost all have a friend or relative with health problems related with morbid obesity and overweight. ${ }^{1,2}$ People usually consider only two possible scenarios in the fight of weight loss, in one hand adopting a healthy life style that includes (routinely physical activity, healthy and balanced food, an appropriated eating habits.); and the second scenario is characterized by expect amazing results in terms of weight loss in a short time period with no major efforts. Frequently patients belonging to this scenario consider surgical alternatives, either cosmetic such as lipectomy, liposuction, and liposculpture or functional including bariatric surgery procedures. Bariatric approaches in the treatment of obesity has a lot of medical evidence, clinical studies with adequate follow up, supporting great clinical outcomes, however there is still a lack of information in the population regarding advantages, limitations, inclusion and exclusion criteria to be considered for this type of procedures. ${ }^{3,4}$

The successful rate of bariatric is high, nonetheless, is also common to find patient in all countries that where expose to bariatric surgery procedures without achieving positive clinical results. Experts in the field state that most those "failed patients" were selected for surgery not following the guidelines proposed by the medical surgical associations and societies. ${ }^{2-5}$ Analyzing the existing obese population around the world, an important percentage of the patients with problems related with healthy food habits elude psychological problems as a way to scape to the reality. This scoop is a very important starting point that must be checked always with each patient. As an interdisciplinary group, the medical teams in the daily clinical practice perform their procedures and activities following the guidelines proposed by medical and academic associations, based on the current clinical evidence available and even in its own clinical experience. ${ }^{3,5}$ Usually, patients fail in their clinical outcomes expected not due to the surgical procedure; there are other factors to consider in this set-up such as: personal care and awareness of the disease, inappropriate diet, lack of physical activity, and absence of emotional support by psychologists or experts in the area. Other medical specialties are supposed to be integrated in the medical treatment of the obese patient. Based on the concepts mentioned above, if patients and their families are committed to taking into account the recommendations and medical indications given by the physicians and healthcare professionals, better results could be found during the follow ups and medical check ups. The relevance given to this part of the treatment is irrelevant or not well valued by patients because they prefer quick results with minimal efforts. ${ }^{6,7}$

Finally, from our perspective, bariatric programs around the world must evaluate all the clinical and mental factors behind the patients
Volume 8 Issue I - 2018

\author{
Patrick F Tarquino,' John E Pedraza, ${ }^{2}$ Juan S \\ Barajas Gamboa ${ }^{3}$ \\ Universidad El Rosario, Facultad de Medicina, Colombia \\ ¿Universidad Pontificia Javeriana, Facultad de Medicina, Colombia \\ ${ }^{3}$ Universidad Autonoma de Bucaramanga, Facultad de Ciencias \\ de la Salud, Colombia
}

Correspondence: Juan S Barajas-Gamboa, Universidad Autonoma de Bucaramanga, Facultad de Ciencias de la Salud, Colombia, Email jbarajasgamboa@gmail.com

Received: January 16, 2018 | Published: February 20, 2018

and their perspectivesprior to consider any surgical procedure for the management of the morbid obesity and comorbidities. Ensuring the patient's commitment to face the different stages of the treatment, would give more chances to obtain better clinical outcomes and avoid medical failures that have high monetary costs for the insurance companies and negative impact in patients. ${ }^{1,4} \mathrm{We}$ invite bariatric groups all over the world, to not underestimate patients and see them as an organic and mental human being.

\section{Acknowledgements}

None.

\section{Conflict of interest}

The author declares no conflict of interest.

\section{References}

1. Al-Mutawa A, Anderson AK, Alsabah S. Nutritional Status of Bariatric Surgery Candidates. Nutrients. 2018;1:10(1).

2. Batsis JA, Zagaria AB. Addressing Obesity in Aging Patients. Med Clin North Am. 2018;102(1):65-85.

3. Ivezaj V, Wiedemann AA, Grilo CM. Food addiction and bariatric surgery: a systematic review of the literature. Obes Rev. 2017;18(12):1386-1397.

4. Adams TD, Davidson LE, Litwin SE, etal. Weight and Metabolic Outcomes 12Years after Gastric Bypass. N Engl J Med. 2017;377(12):1143-1155.

5. Barajas-Gamboa JS, Nino DF. Adolescent and Children Obesity Surgery: Current Trends and Future Perspectives. Adv Obes Weight Manag Control. 2015;3(5):00071.

6. Puia A, Puia IC, Cristea PG. Ethical considerations in bariatric surgery in a developing country. Clujul Med. 2017;90(3):268-272.

7. Lee PC, Dixon J. Bariatric-metabolic surgery: A guide for the primary care physician. Aust Fam Physician. 2017;46(7):465-471. 